\title{
RESEARCH PAPER \\ A MULTI-PERIOD MARKOV MODEL FOR MONTHLY RAINFALL IN LAGOS, NIGERIA
}

\author{
K. O. Aiyesimoju and A. O. Busari \\ Department of Civil and Environmental Engineering \\ University of Lagos, Akoka-Yaba, Lagos, Nigeria

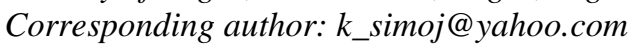

\begin{abstract}
Long periods of historical hydrological data such as rainfall and streamflow which are necessary for planning and design of water resources projects, are often not available and have to be forecasted. Many models available for this were developed and tested in developed countries in temperate climates and so their application in tropical climates is questionable. A twelve-period Markov model has been developed for the monthly rainfall data for Lagos, along the coast of South Western Nigeria. The goodness of fit of the model was assessed by estimating the autocorrelations of the residuals of the historical data (from January 1924 to December 1983) for lags one to sixty. A $95 \%$ confidence band was also established for the autocorrelations. The results show that all but two of the autocorrelations fall within the $95 \%$ confidence band confirming that the residuals are indeed white noise. This indicates that the model is very adequate.
\end{abstract}

Keywords: Markov, multi-period,rainfall model

\section{INTRODUCTION}

The problem of inadequacy or sometimes a total lack of stream flow data if not resolved would definitely lead to an underestimation of water resources facilities or their overestimation. This is why the first attempt to solve the problem of short record length was as far back as the beginning of the $20^{\text {th }}$ century. Hazen (1914) suggested combining records from several stations whilst Sudler (1927) on the other hand wrote historic records on cards and randomly picked the cards, with replacement, to generate 1000 years record. These approaches have the shortcoming of being unable to generate flows outside the actual historical range. This is serious because the potential for large and complex variation over time of hydrological data (see Koutsoyinannis and Montanori, 2007) may result in future flows well outside of the historically observed range.

In reservoir size estimation, the required capacity of a reservoir depends on the sequence of inflows into it versus the sequence of required 


\section{Aiyesimoju and Busari}

withdrawals from it. In this case, it is not so important to know the exact sequence of future inflows. All that is needed is to generate future inflows with the same stochastic properties as the historical record. This is why stochastic models were introduced in hydrology to generate inflows that have equal probability of occurrence as the historical values.

\section{RELATED WORKS}

Stochastic models were pioneered by Barnes (1954) and Thomas and Fiering (1962). Box and Jenkins (1972) generalized existing models and further developed them. The resulting BoxJenkins models; namely Autoregressive (AR), Moving Average (MA), and their combinations greatly popularized stochastic modeling despite their shortcomings which include short range dependence, parsimony problems and a lack of physical meaning especially for the higher order models. These shortcomings led to the development of other models such as Fractional Gaussian Noise Processes (Mandelbrot, 1965), Broken Line Processes (Mejia et al., 1972), Disaggregation Models (Valencia and Schaake, 1973), etc. More recently Artificial neural Network (ANN) models (Abrahart et al, 2007; Birikundavyl et al., 2002; Corzo and Solomatine, 2005; De Vos and Rietjes, 2007, Kant et al. 2013, Borga et al., 2011, Chen et al., 2015) which are connectionist in nature, have been developed. Also, advances in non-linear dynamical systems (Langousis and Koutsoyinannis, 2006, Leedal et al., 2013) have led to the development of models which possess a deterministic basis. These latest models are however data driven and of a black box nature thus providing no process insight. Worse still, they do not provide tools for Monte Carlo simulation which is a serious problem as the whole point of stochastic simulation is to extend the record. Several other authors focused on the effect of uncertainty on the predictions of hydrological phenomena e.g. Weerts et al. (2011) and Zappa et al. (2011). More recently, Ursu and Pereau (2015) utilized a periodic autoregressive process to model river flow; Deo et al. (2015) utilized an adaptive regression spline model to estimate monthly evaporative loss; Pinto et. al (2015) compared models for forecasting monthly streamflows; but all of them were developed in a different environment from tropical Africa.

Selection of a suitable model among the numerous options available may not be clear but factors that will influence the suitability of a model are the nature of the physical processes involved, the quality of the data available, environmental factors such as climate, and, the use to be made of the model. The aim of this work is to develop a multi-period Markov model for monthly rainfall forecasting using monthly rainfall data for Lagos. The model will be assessed by checking if the autocorrelations at different lags of the residuals of the model when applied to the historical data are statistically insignificant. This will confirm the suitability of such a model for stochastic simulation of monthly rainfall data in our tropical and coastal environment. Monthly rainfall is well known to exhibit a seasonality of twelve months and thus twelve periods would be the natural choice and is adopted here.

\section{Periodic Markov Model}

The standard Markov Model is based on the premise that the variate at a particular time is linearly dependent on that at the immediately preceding time plus a random component as follows:

$X_{i}=r X_{i-1}+a_{i}$

where $X_{i}$ is the variate value at time $i, a_{i}$ is random deviate at time $i$ and $r$ is the lag one autocorrelation coefficient for series $X_{i}$. Taking the expectation and variance of Equation (1) implies

$$
\begin{aligned}
& E\left(X_{i}\right)=r E\left(X_{i-1}\right)+E\left(a_{i}\right) \\
& \operatorname{Var}\left(X_{i}\right)=\operatorname{Var}\left(r X_{i-1}\right)+\operatorname{Var}\left(a_{i}\right) \\
& =r^{2} \operatorname{Var}\left(X_{i-1}\right)+\operatorname{Var}\left(a_{i}\right)
\end{aligned}
$$

Assuming stationarity, then $E\left(X_{i}\right)=E\left(X_{i-1}\right)=\mu_{X}$ 
$\operatorname{Var}\left(X_{i}\right)=\operatorname{Var}\left(X_{i-1}\right)=\sigma_{X}^{2}$ and let $E\left(a_{i}\right)=\mu_{a}$

or

and $\operatorname{Var}\left(a_{i}\right)=\sigma_{a}^{2}$

Then from Equations (2) and (3)

$\mu_{a}=\mu_{X}(1-r)$

$\sigma_{a}^{2}=\left(1-r^{2}\right) \sigma_{X}^{2}$

Assuming our variates are normally distributed and if $t_{i}$ represents a standard normal random deviate at time $i$, then

$a_{i}=\mu_{a}+t_{i} \sigma_{a}=\mu_{X}(1-r)+t_{i} \sigma_{X} \sqrt{\left(1-r^{2}\right)}$

Dropping the subscript $X$, Equation (1) can be written in terms of the parameters of the original variate $X$ as

$X_{i}=r X_{i-1}+\mu(1-r)+t_{i} \sigma \sqrt{\left(1-r^{2}\right)}$

which can be written as

$\frac{X_{i}-\mu}{\sigma}=r \frac{X_{i-1}-\mu}{\sigma}+t_{i} \sqrt{\left(1-r^{2}\right)}$

Equation (8) in effect shows that prior normalization of the deviates simplifies the model.

Hydrological data however exhibit an annual period. Thus for monthly rainfall that is of interest here, this translates to a period of twelve months. In Equation (8), the variate for a time $i$ depends on only the variate for the previous time $i-1$ and a random component. However, seasonality implies that the dependence of the variates belonging to period $j+1$ on their immediately preceding values (period $j$ ) is different from the dependence of the variates belonging to period $j$ on their immediately preceding values (period $j-1$ ). Let $j(i)$ be the period to which time $i$ belongs, a multi-period Markov model can thus be written as

$$
\frac{X_{i}-\mu_{j(i)}}{\sigma_{j(i)}}=r_{j(i)} \frac{X_{i-1}-\mu_{j(i-1)}}{\sigma_{j(i-1)}}+t_{i} \sqrt{\left(1-r_{j(i)}^{2}\right)}
$$

$x_{i}=r_{j(i)} x_{i-1}+t_{i} \sqrt{\left(1-r_{j(i)}{ }^{2}\right)}$

where $x_{i}$ is the normalized $X_{i}$ as follows

$x_{i}=\frac{X_{i}-\mu_{j(i)}}{\sigma_{j(i)}}$

Note above, $r_{j}$ is the autocorrelation of the normalized variates belonging to period $j$ to those immediately preceding them (period $j-1$ ). Index $i$ is consecutively numbered from 1 to total no of data whilst index $j$ will vary from 1 to number of periods, which is twelve here. For example, $j$ for all January values will be 1 whilst that for all December values will be 12. $\mu_{j}$ and $\sigma_{j}$ are ideally the population mean and standard deviation respectively for period $j$ values. Let $i \in j$ represent only $i$ values belonging to period $j$, the sample estimates used were as follows

$\mu_{j}=\frac{\sum_{i \in j} X_{i}}{T_{j}}$

$\sigma_{j}=\sqrt{\frac{\sum_{i \in j}\left(X_{i}-\mu_{j}\right)^{2}}{T_{j}}}$

$r_{j}=\frac{\frac{1}{T_{j}} \sum_{i \in j}\left(X_{i}-\mu_{j}\right)\left(X_{i-1}-\mu_{j-1}\right)}{\sigma_{j} \sigma_{j-1}}$

or

$r_{j}=\frac{\sum_{i \in j} x_{i} x_{i-1}}{T_{j}}$

where $T_{j}$ is the number of values in the summation belonging to period $j$. Equations (10), (11) and (12a) are used to estimate the parameters of the model.

GOODNESS OF FIT MODEL

After the parameters of the model have been determined, application of the model to the 


\section{Aiyesimoju and Busari}

historical data will not show a perfect fit. The resulting errors are termed as residuals and they represent the random components corresponding to the historical data. For a good model, the residuals should be white noise in which case, their autocorrelation $\rho_{j}$ at any lag $j$ should be zero. However, since only a sample is available here, the $100(1-\alpha) \%$ confidence interval for the sample autocorrelation $\rho_{j}$ at any lag $j$, is (Yevjevich, 1972)

$\frac{-t(T-2, \alpha / 2)}{\left(T-2+t^{2}(T-2, \alpha / 2)\right)^{1 / 2}} \leq \rho_{j} \leq \frac{t(T-2, \alpha / 2)}{\left(T-2+t^{2}(T-2, \alpha / 2)\right)^{1 / 2}}$

where $T$ is the sample size and $t(N, \alpha)$ is a student-t random deviate with $N$ degrees of freedom and a probability of exceedance of $\alpha$. The residuals $\hat{t}_{i}$ of the historical data are obtained by simply replacing the random component in Equation (9a) or (9b) with the residual $\hat{t}_{i}$ to give

$\frac{X_{i}-\mu_{j(i)}}{\sigma_{j(i)}}=r_{j(i)} \frac{X_{i-1}-\mu_{j(i-1)}}{\sigma_{j(i-1)}}+\hat{t}_{i} \sqrt{\left(1-r_{j(i)}{ }^{2}\right)}$

This implies

$\hat{t}_{i}=\frac{\frac{X_{i}-\mu_{j(i)}}{\sigma_{j(i)}}-r_{j(i)} \frac{X_{i-1}-\mu_{j(i-1)}}{\sigma_{j(i-1)}}}{\sqrt{\left(1-r_{j(i)}^{2}\right)}}$

or

$\hat{t}_{i}=\frac{x_{i}-r_{j(i)} x_{i-1}}{\sqrt{\left(1-r_{j(i)}^{2}\right)}}$

\section{RESULTS AND DISCUSSION}

Monthly rainfall data from January 1924 to
South Western Nigeria (Table 1) were obtained, making a total of 720 data points. The parameters of the model estimated from the historical data using Equations (10), (11) and (12a) are as shown in Table 2. To test the goodness of fit, the residuals (model errors) for the historical data were obtained using Equation (14a). The autocorrelation values of these residuals for lags one to sixty are plotted in Fig. 1. The $95 \%$ confidence band for these from Equation (13) is also plotted. This shows that all but two fall within the $95 \%$ confidence band which is very good. Furthermore, 1000 years of data were generated with the model (using Equation (9a)). The parameters obtained for them are shown in Table 3 and they compare very well with Table 2 , further validating the model.

\section{CONCLUSION}

A twelve-period Markov stochastic model has been developed for the monthly rainfall data for Lagos along the coast of South Western Nigeria. The goodness of fit of the model was assessed by estimating the autocorrelations of the residuals of the historical data (from January 1924 to December 1983) for lags one to sixty. A 95\% confidence band was also established for the autocorrelations. The results show that all but two of the autocorrelations fall within the $95 \%$ confidence band, confirming that the residuals are indeed white noise. This indicates that the model is very adequate. This confirms the suitability of such a model for stochastic simulation of monthly rainfall data in our tropical and coastal environment. An important limitation of the model is the fact that theoretically, negative values are possible which is practically meaningless. Thus in practice, negative values are treated as zeros. 


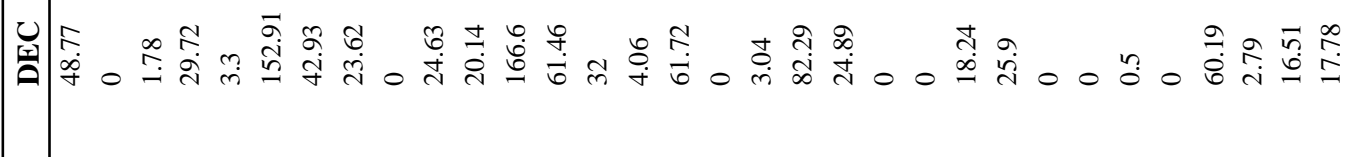

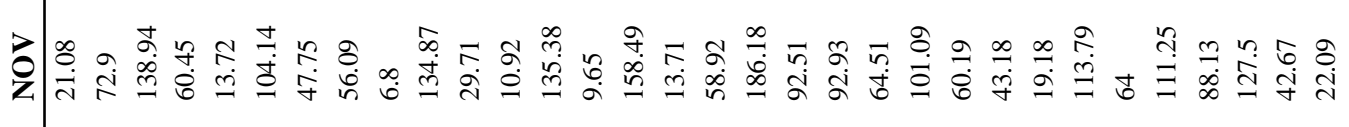

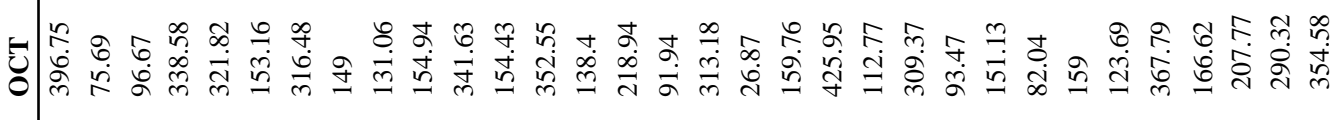

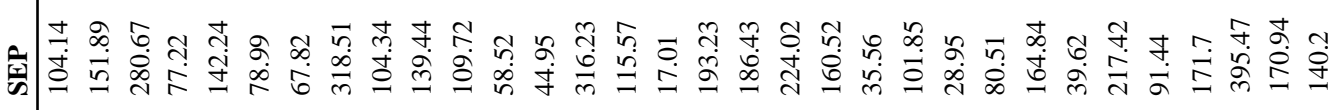

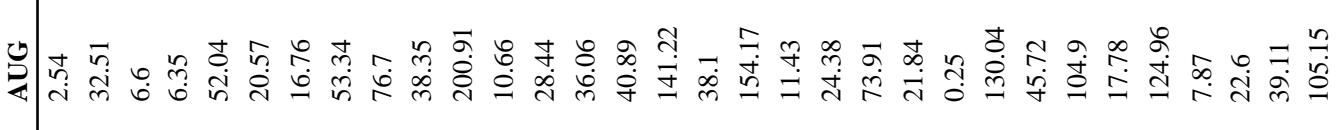

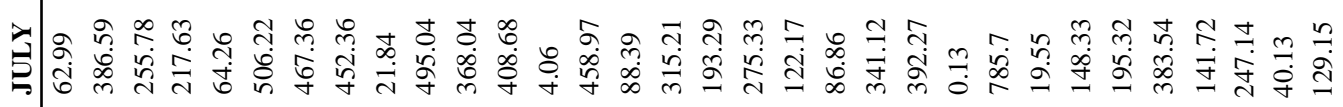

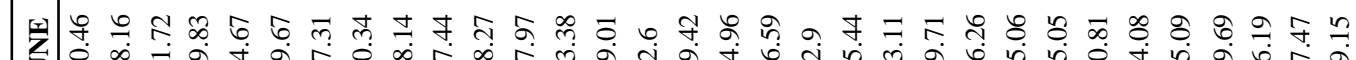

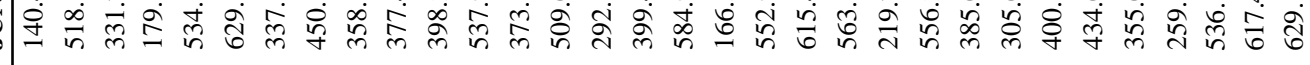

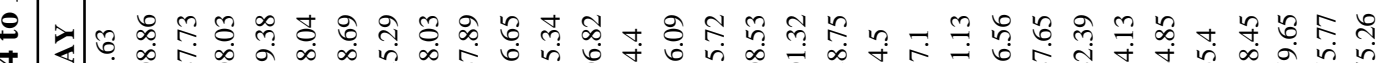

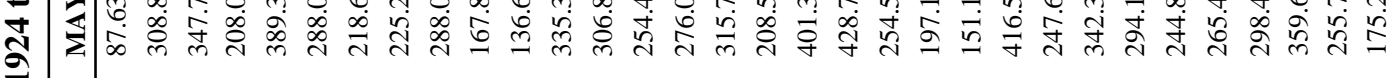

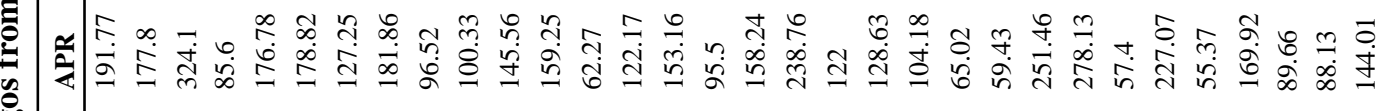

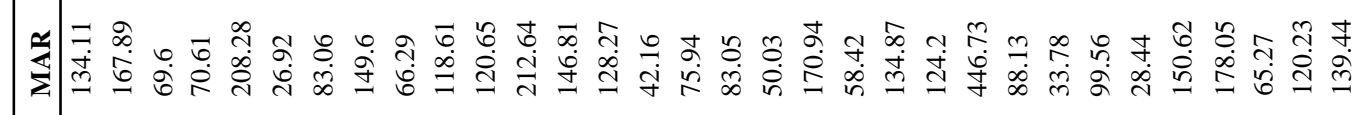

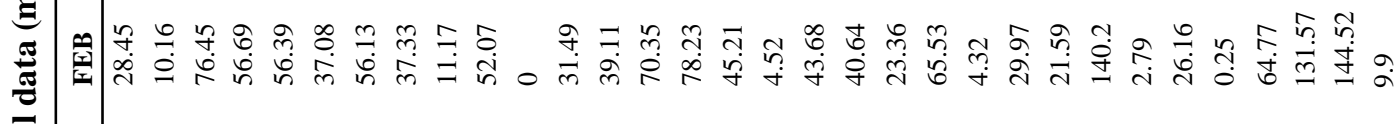

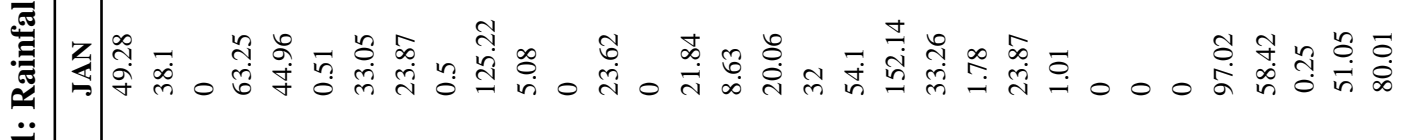

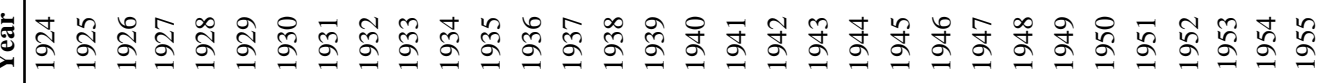




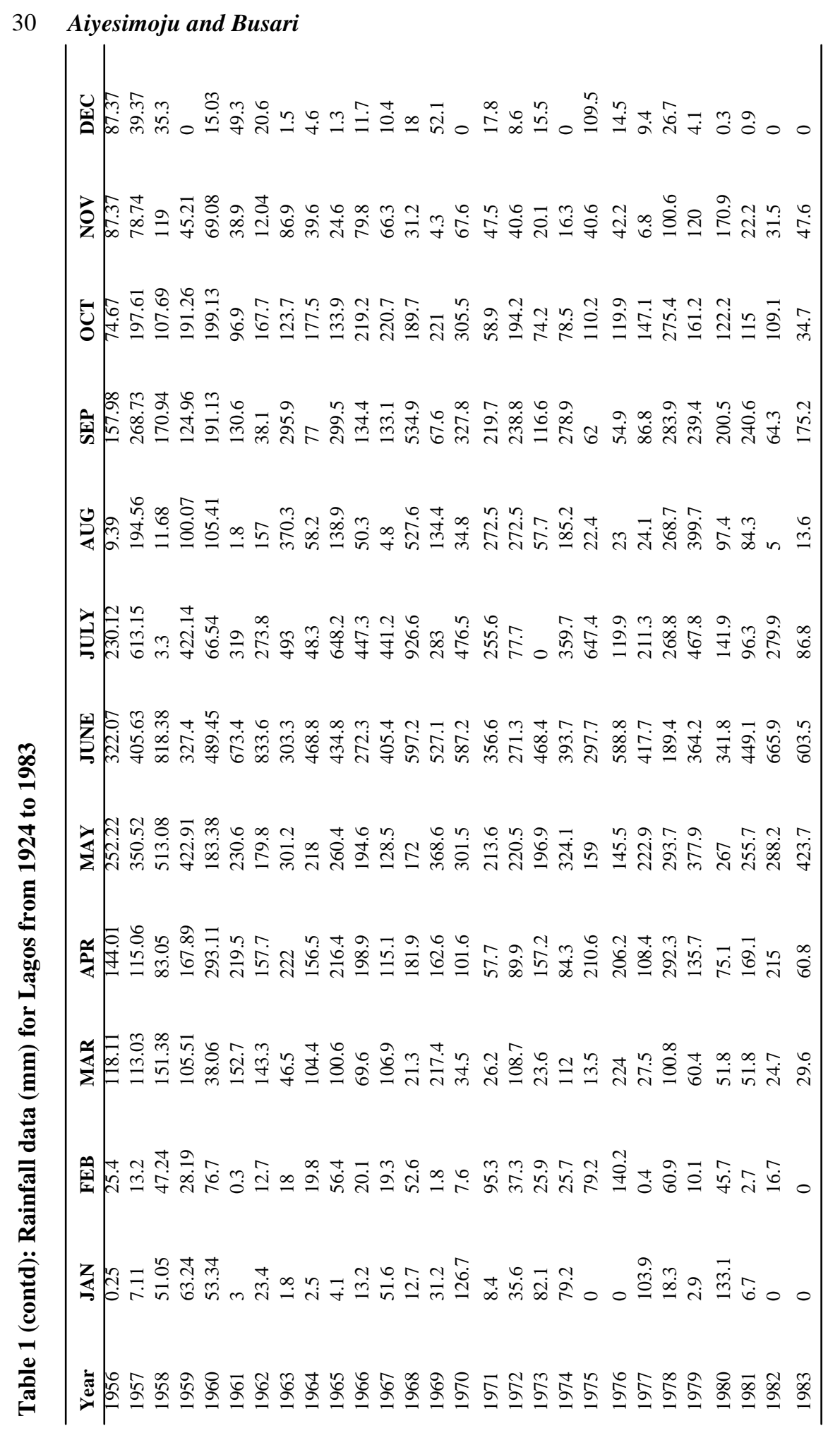

Journal of Science and Technology (C) KNUST December 2015 


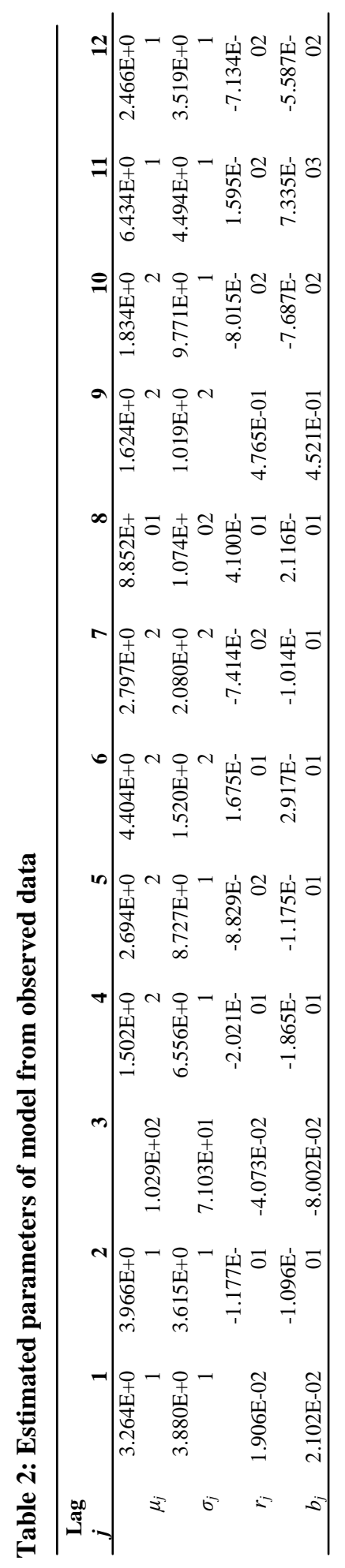

Multi-period Markov model for rainfall in Lagos ...

31

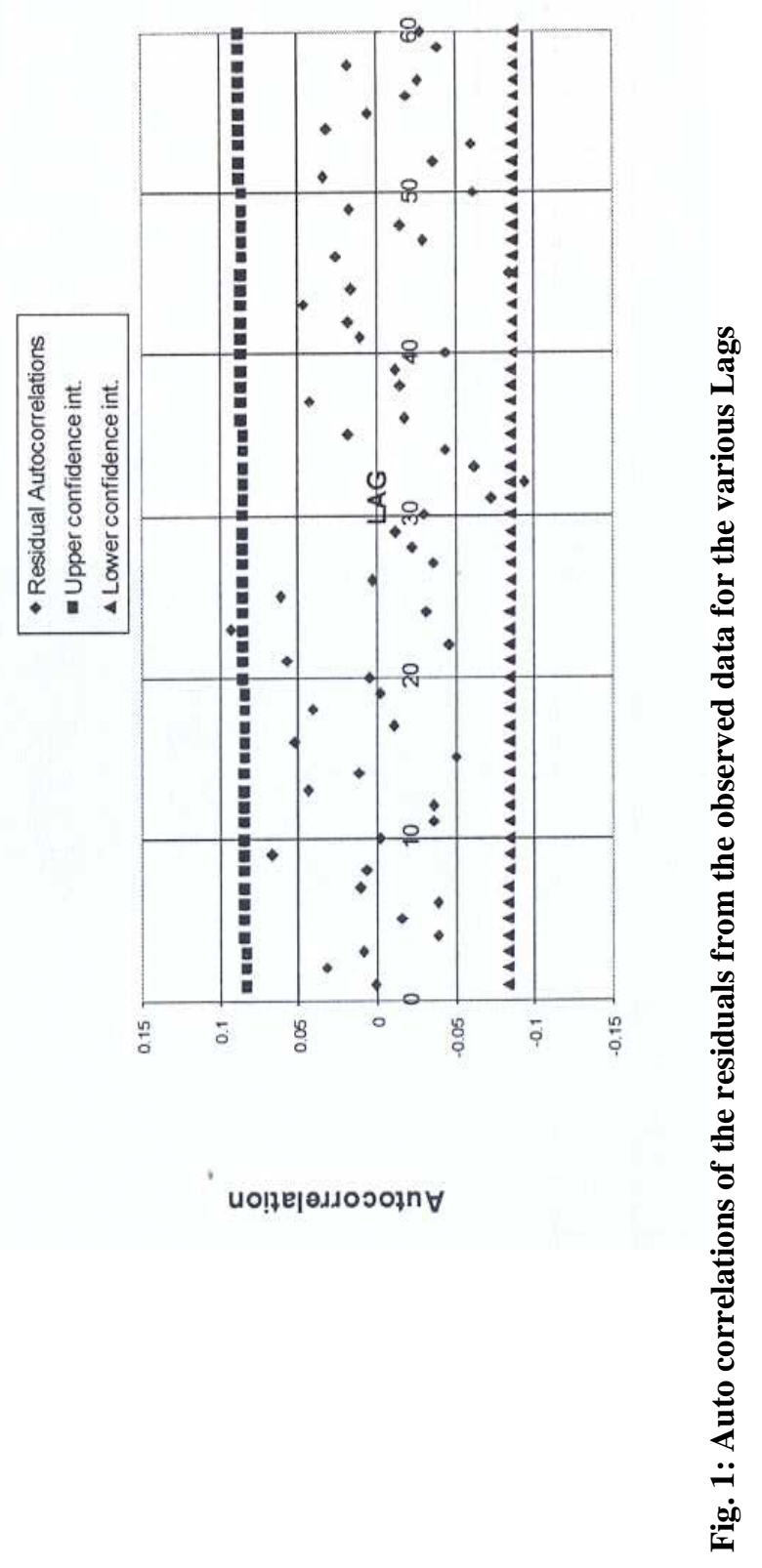

Journal of Science and Technology @ KNUST December 2015 


\section{REFERENCES}

Abrahart, R. J., Heppenstall, A. J. and See, L. N. (2007). Timing error correction procedure applied to neural network rainfall-runoff modeling. Hydrological Sciences Journal, 52(3): $414-431$.

Barnes, F. B. (1954). Storage required for a city water supply. Journal of Institution of Engineers, Australia. 26(9): 198-203.

Birikundavyl, S., Labid, R., Trung, H. and Rousselle, J. (2002). Performance of Neural Networks in Daily Streamflow Forecasting. Journal of Hydrologic Engineering, 7(5):392 -398 .

Borga, M., Anagnostou, E. N., Blöschl, G., Creutin, J. D. (2011). Flash flood forecasting, warning and risk management: the HYDRATE project, Environmental Science and Policy, 14(7):834-844

Box, G. E. P. and Jenkins, G. M. (1972). Time series analysis, forecasting and control. Holden Day Inc., San Francisco

Chen, X. Y., Chau, K. W. and Busari A. O. (2015). A comparative study of populationbased optimization algorithms for downstream river flow forecasting by a hybrid neural network model. Engineering Applications of Artificial Intelligence, 46(A): 258268

Corzo, G. S. and Solomatine, D. (2007). Baseflow separation technique for modular artificial neural network modeling in flow forecasting. Hydrological Sciences Journal, 52 (3): 491-507.

De Vos, N. J. and Rietjes, T. H. M. (2007). Multi-objective performance comparison of an artificial neural network and a conceptual rainfall-runoff model. Hydrological Sciences Journal, 52(3): 397-413.

Deo, R. C., Samui, P. and Kim, D. (2015). Esti- 
mation of monthly evaporative loss using . relevance vector machine, extreme learning machine and multivariate adaptive regression spline models. Stochastic Environmental Research and Risk Assessment, DOI 10.1007/ s00477-015-1153-y. , pp. 1-16

Hazen, A. (1914). Storage to Be Provided in Impounding Reservoirs for Municipal Water Supply. Trans. ASCE, .77: 1539-1669

Kant, A., Suman, P. K., Giri, B. K., Tiwari, M. K., Chatterjee, C., Nayak, P. C. and Kumar, S. (2013). Comparison of multi-objective evolutionary neural network, adaptive neurofuzzy inference system and bootstrap-based neural network for flood forecasting, Neural Computing and Applications,23(Suppl 1):S231-S246

Langousis, A. and Koutsoyinannis, D. (2006). A stochastic methodology for generation of seasonal time series reproducing overheard scaling. Journal of Hydrology, 322(48):138154.

Leedal, D., Weerts, A. H., Smith, P. J. and Beven, K. J. (2013). Application of databased mechanistic modelling for flood forecasting at multiple locations in the Eden catchment in the National Flood Forecasting System (England and Wales), Hydrology and Earth System Sciences, 17:177-185

Koutsoyinannis, D. and Montanori, A. (2007). Statistical analysis of hdyroclimatic time series: uncertainty and insights. Water Resources Research, 43(5): 5429:1-9.

Mandelbrot, B. B. (1965). Une Class de processes stochastic ques jomothetique a soi! Application a la loi climatologique. Compte Rendus, Academic Science. 260:3284-3277.

Mejia, J. M., Rodriguez-Iturbe, I. and Dawdy, D. R. (1972). Streamflow Simulation; The Broken Line Process as a Potential Model for Hydrologic Simulation. Water Resources
Research, 8(4):931-941

Pinto, W. P., Lima, G. B. and Zanetti, J. B. (2015). Comparative Analysis of Models for Times to Series Modeling and Forecasting of Scheme of Average Monthly Streamflow of the Doce River, Colatina Espirito Santo, Brazil. Ciência e Natura, 37(4): 1-11

Sudler, C. E. (1927). Storage Required for the Regulation of Streamflow. Trans. ASCE, 91: 622-704

Thomas, H. A. S. and Fiering, M. B. (1962). Mathematical Synthesis of streamflow sequences for the analysis of river basins by simulation. In Design of Water Resources Systems, by A Maass, et al. Harvard University Press, Cambridge, Mass.

Ursu, E. and Pereau J. C. (2015). Application of periodic autoregressive process to the modeling of the Garonne river flows. Stochastic Environmental Research and Risk Assessment. DOI 10.1007/s00477-015-11933., pp. 1-11

Valencia, D. and Schaake, Jr. J. C. (1976). Disaggregation process in stochastic hydrology. Water Resources Research, 9:580-585

Weerts, A. H., Winsemius, H. C. and Verkade, J. S. (2011). Estimation of predictive hydrological uncertainty using quantile regression: examples from the National Flood Forecasting System (England and Wales), Hydrology and Earth System Science, 15(1):255-265

Yevjevich, V. (1972). Probability and Statistics in Hydrology. Water Resources Publications, Fort Collins, Colorado

Zappa, M., Jaun, S., Germann, U., Walser, A. and Fundel, F. (2011). Superposition of three sources of uncertainties in operational flood forecasting chains, Atmospheric Research, 100(2\&3):246-262 